\title{
Urine sample used for detection of Toxoplasma gondii infection by loop-mediated isothermal amplification (LAMP)
}

\author{
Xin Hu${ }^{1}$, Chang-Wang Pan ${ }^{2}$, Ya-Fei Li ${ }^{1}$, Han Wang ${ }^{2}$ and Feng Tan ${ }^{2}$ \\ ${ }^{1}$ School of Medical Laboratory Science and School of Life Science, Wenzhou Medical College, Wenzhou, Zhejiang 325035 , \\ P. R. China; \\ ${ }^{2}$ Department of Parasitology, School of Basic Medical Sciences, Wenzhou Medical College, Wenzhou, Zhejiang 325035, \\ P. R. China
}

\begin{abstract}
In this study, a loop-mediated isothermal amplification (LAMP) assay was established to detect Toxoplasma gondii DNA in mice infected with $T$. gondii PRU strain. This LAMP assay was based on the sequence of highly repetitive B1 gene. The detection limit of $T$. gondii LAMP assay was $1 \mathrm{pg}$ of $T$. gondii DNA, which was evaluated using 10-fold serially diluted DNA of cultured parasites. The LAMP assay was also highly specific for $T$. gondii and able to detect $T$. gondii DNA in urine of mice treated with dexamethasone at 90 day post infection (p.i.), although this assay could not detect the DNA in mice urine 2-6 days p.i. These results demonstrated that LAMP is effective for evaluation of therapy effectiveness for T. gondii infection. The established LAMP assay may represent a useful and practical tool for the routine diagnosis and therapeutic evaluation of human toxoplasmosis.
\end{abstract}

Keywords: Toxoplasma gondii, toxoplasmosis, loop-mediated isothermal amplification (LAMP), urine, B1, mouse

Toxoplasmosis, a disease caused by the parasitic protozoan Toxoplasma gondii (Nicolle et Manceaux, 1908) is distributed worldwide. Infected immunocompetent individuals present only mild symptoms or may even be completely asymptomatic. Nevertheless, in congenitally infected children and immunocompromised persons such as human immunodeficiency virus-infected individuals, organ transplant recipients, and cancer patients, infection causes high rates of morbidity and mortality (Zangerle et al. 1991, Germann and Matter 1994). Thus, the availability of a sensitive and reliable means of diagnosis early after infection is crucial, because any delay in appropriate treatment can result in irreversible damage.

The alarming incidence of toxoplasmic encephalitis in patients with acquired immunodeficiency syndrome and the difficulty encountered in establishing the diagnosis without brain biopsy have served as an impetus for development of non-invasive methods for diagnosis of this entity (Luft and Remington 1988).

The standard diagnostic methods of toxoplasmosis are based on serology. Unfortunately, their results are very difficult to interpret for immunocompromised patients, foetuses and infants. Recently, methods for genetic diagnosis of toxoplasmosis using PCR have been developed. Although PCR-based assays have been demonstrated to provide reliable, sensitive and specific information, these PCR techniques are not in widespread use in the resource- poor countries due to the relatively complex nature of the assays, and the need for expensive equipment and welltrained personnel.

Toxoplasma antigens have been demonstrated in urine from mice acutely infected with $T$. gondii as well as from human patients with acute toxoplasmic encephalitis (Huskinson et al. 1989). Tachyzoites of T. gondii were also identified in urine samples (Fuentes et al. 1996, Nguyen et al. 1996). However, these methods were rather elaborate and expensive, and thus not suitable for routine examination in the developing countries. Therefore, the development of cost-effective, simple and rapid detection methods is still needed for the diagnosis of toxoplasmosis.

A novel nucleic acid amplification method, loop-mediated isothermal amplification (LAMP), which amplifies DNA with high specificity, efficiency, simplicity and rapidity under isothermal conditions, has been developed (Notomi et al. 2000). This method does not entail the use of expensive equipment. Furthermore, LAMP enables the amplification of a few copies of DNA to attain $10^{9}$ copies in less than $1 \mathrm{~h}$ under isothermal conditions and the amplification products can easily be observed by the naked eye. The use of four LAMP primers, which are designed to recognize six distinct regions on the target gene, assures specific amplification. LAMP has been developed for detection of various parasites, including Plasmodium falciparum (see Poon et al. 2006), African trypanosomes 
(Njiru et al. 2008), Babesia (Guan et al. 2008), Pneumocystis (Uemura et al. 2008) and T. gondii (Krasteva et al. 2009). As a result, LAMP has been reported to be a highly sensitive and specific method for the detection of parasitic infections. Moreover, LAMP is an attractive diagnostic method in resource-poor countries.

We describe here a LAMP method, based on B1 gene of the T. gondii PRU strain, developed as a rapid, sensitive and convenient method for detection of $T$. gondii. Furthermore, we evaluated its feasibility in the urine samples collected from mice experimentally infected with the T. gondii PRU strain.

\section{MATERIALS AND METHODS}

\section{Parasite culture and DNA extraction}

The brain cyst-forming PRU strain tachyzoites were propagated in vitro under standard procedures, by serial passages in COS-7 monolayers in Dulbecco's modified Eagle medium supplemented with $10 \%$ heat-inactivated foetal bovine serum at $37{ }^{\circ} \mathrm{C}$ and under $5 \% \mathrm{CO}_{2}$. Tachyzoites were collected by scraping the cell monolayer, released by passage through a 26 -gauge needle, then filtered on a $3 \mu \mathrm{m}$ polycarbonate membrane filter to remove host-cell debris. They were pelleted at $750 \mathrm{~g}$ for $10 \mathrm{~min}$ and washed twice with PBS. The purified parasites were resuspended in a DNA extraction buffer and DNA was extracted with a Universal Genomic DNA Extraction Kit (Takara) according to the manufacturer's instructions. Genomic DNA of COS-7 was used as a negative control.

\section{LAMP reaction}

The LAMP reaction was conducted according to the method previously described (Yang et al. 2008). Briefly, specific LAMP primer set consisting of primers F3 (5'-ACC AGC AGC AGA GGA GTG -3'), B3 (5'-CCA TCA GAC GAA TCA ACG GA3'), FIP (5'-CCT CCT CTT CGC GAA ACC TCA AAG ATG CCT AGA GGA GAC ACA-3'), BIP (5'-AAC AAG AGA CGT GCC GCA TGT ACT GTG CCA TTT TCT GAG CA-3') was designed by using the PrimerExplorer V3 program to amplify the T. gondii B1 gene (GenBank accession no. AF179871). LAMP reaction mixture contained $1.6 \mu \mathrm{M}$ each of primers FIP and BIP, $0.2 \mu \mathrm{M}$ each of primers $\mathrm{F} 3$ and $\mathrm{B} 3,1.4 \mathrm{mM}$ of dNTP

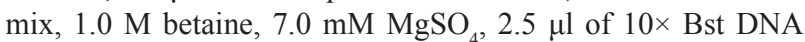
Polymerase Buffer, $8 \mathrm{U}$ of Bst DNA polymerase, along with $1 \mu \mathrm{l}$ of DNA template, in a final volume of $25 \mu \mathrm{l}$. The reaction mixture was incubated in a heat block at $63^{\circ} \mathrm{C}$ for $60 \mathrm{~min}$ and then inactivation was carried out at $80^{\circ} \mathrm{C}$ for $2 \mathrm{~min}$. To ensure that the loop primers did not induce self-amplification, a control reaction was carried out in the absence of any template.

\section{Detection of LAMP product}

The LAMP products were loaded on $2 \%$ agarose gel for electrophoresis and visualized and photographed under UV light after staining with ethidium bromide. LAMP-positive results are characterized by the appearance of ladder-like pattern on agarose gel, with many bands of different sizes up to the loading well.

Visual inspection of the LAMP amplicons in the reaction tube was performed by adding SYBR Green I fluorescent dye after the inactivation of the reaction tubes; the fluorescent signals of the solutions were observed by the naked eye. A colour change from orange to green was observed for positive reactions.

\section{PCR specificity of LAMP outer primers}

To confirm that the LAMP amplified the correct target, PCR was performed with two outer primers, F3 and B3, to amplify the LAMP products. The PCR assay was conducted in $20 \mu \mathrm{lre}$ action mixture containing $10 \mu \mathrm{l}$ of $2 \times$ PCR Master Mix, $0.5 \mu l$ of $20 \mathrm{pmol} / 1 \mathrm{~F} 3$ and B3, and $1 \mu \mathrm{LAMP}$ products. Initial denaturation was conducted at $94^{\circ} \mathrm{C}$ for $3 \mathrm{~min}$, followed by 35 cycles of denaturation $\left(60 \mathrm{~s}\right.$ at $\left.94^{\circ} \mathrm{C}\right)$, annealing $\left(60 \mathrm{~s}\right.$ at $\left.55^{\circ} \mathrm{C}\right)$ and extension $\left(60 \mathrm{~s}\right.$ at $\left.72^{\circ} \mathrm{C}\right)$. Three microlitres aliquots were subjected to $2 \%$ agarose gel electrophoresis, stained with ethidium bromide, and visualized under UV light.

\section{Specificity and sensitivity of LAMP}

The specificity of the Toxoplasma gondii LAMP primers was determined by testing them against DNA derived from Pneumocystis jiroveci, Trichomonas vaginalis, Acanthamoeba sp., and Cryptosporidium parvum. The sensitivity of the assay was confirmed using genomic DNA, which was serially diluted to contain an amount of DNA equivalent to a range of $1-10,000$ tachyzoites per LAMP reaction.

Application of LAMP assays in urine samples collected from infected mice

Ten female ICR mice 6-8 weeks old were purchased from Wenzhou Medical College Laboratory Animal Center in China. Urine samples were collected for $24 \mathrm{~h}$ and used as negative control. Thereafter, mice were infected orally with 20 PRU tissue cysts and urine samples were collected on days 2 through 6 post infection (p.i.) and stored at $-20^{\circ} \mathrm{C}$. Urine samples from each mouse collected on a given day were pooled and concentrated by centrifugation at $1,800 \mathrm{~g}$ for $10 \mathrm{~min}$. These pellets were washed 3 times and resuspended in a DNA extraction buffer and DNA was extracted. Then, DNA of urine samples was detected by established LAMP. DNA from tachyzoites of the PRU strain of $T$. gondii was used as a positive control.

Ninety days after infection, dexamethasone (dexamethasone 21-phosphate disodium salt; Sigma) was dissolved at a concentration of $40 \mathrm{mg} / \mathrm{l}$ drinking water in order to suppress the immune system and reactivate $T$. gondii as described previously (Saeij et al. 2008). Five mice uninfected with $T$. gondii were immunosuppressed by dexamethasone and used as a negative control. Subsequently, urine samples were collected on days 5 through 10 after treatment and DNA of urine was extracted and detected by LAMP as described above. All of urine samples from negative control group were pooled to extract total DNA after treatment.

\section{RESULTS}

\section{Detection and confirmation of LAMP product}

Positive results were visually observed in tubes which contained LAMP product of the Toxoplasma gondii PRU strain (Fig. 1). These visual products demonstrated typical patterns of ladder-like bands on agarose gels (Fig. 2). After PCR was used to amplify these LAMP products, an expected 212 bp fragment was obtained (Fig. 3), which is considered to correspond to B1 sequence of $T$. gondii (data not shown). 


\section{Specificity and sensitivity of LAMP}

The LAMP was tested for its specificity using heterogeneous DNA samples as controls. Only the target DNA of $T$. gondii PRU strain was amplified. No cross-reaction was found with DNA samples of Pneumocystis jiroveci, Trichomonas vaginalis, Acanthamoeba sp., and Cryptosporidium parvum (Fig. 2).

The genomic DNA of PRU strain tachyzoites was quantified from $1 \mathrm{ng}$ down to $100 \mathrm{fg}$ by 10 -fold serial dilutions and used to evaluate the sensitivity of $T$. gondii LAMP assay. As shown in Fig. 4, the minimum amount of DNA detectable using the LAMP assay was $1 \mathrm{pg}$.

\section{Application of LAMP assays in urine samples collected from infected mice}

Ten mice were orally infected with $T$. gondii PRU. Urine samples from each mouse were collected at day 2-6 p.i. and checked for the presence of this parasite by LAMP. However, no LAMP-positive samples were obtained (data not shown), after which all mice, except five, died on days between 8 and 15 p.i. We therefore immunosuppressed the surviving mice that were infected with PRU by adding dexamethasone to the drinking water 90 days after the initial infection. As shown in Fig. 5, the parasite was detected in urine samples from five surviving mice by LAMP method.

\section{DISCUSSION}

The accurate diagnosis of toxoplasmosis is extremely important for the control of the disease. Normally, diagnosis can be made by a number of different methods, including the indirect fluorescent-antibody test (IFAT) (Jannuzzi et al. 1981), enzyme-linked immunosorbent assays (ELISA) (Mondesire et al. 1981), tissue culture, and PCR (Homan et al. 2000; Filisetti et al. 2003).

Routine diagnosis of Toxoplasma gondii infection relies mainly on serology (Remington et al. 1995). However, in spite of the high sensitivity, serological methods have some limitations because specific antibodies and $T$. gondii antigens may not be present early in the infection, especially in patients with underlying disease causing suppressed antibody response, patients having immunosuppressive therapy (Ruskin and Remington 1976, Krick and Remington 1978) and certain cases of congenital toxoplasmosis (Stagno 1980). Moreover, antibodies, particularly IgM, may not arise during reactivation of the encysted form of the parasite (Ashburn et al. 1998). Alternatively, the detection of Toxoplasma DNA in amniotic fluids, tissues, or blood by PCR has made a significant improvement in both the prenatal diagnosis of congenital toxoplasmosis and the detection of reactivation in immunocompromised patients.

Several PCR-based techniques have periodically been developed for the diagnosis of toxoplasmosis using various clinical specimens (Johnson et al. 1993, Lee et al.

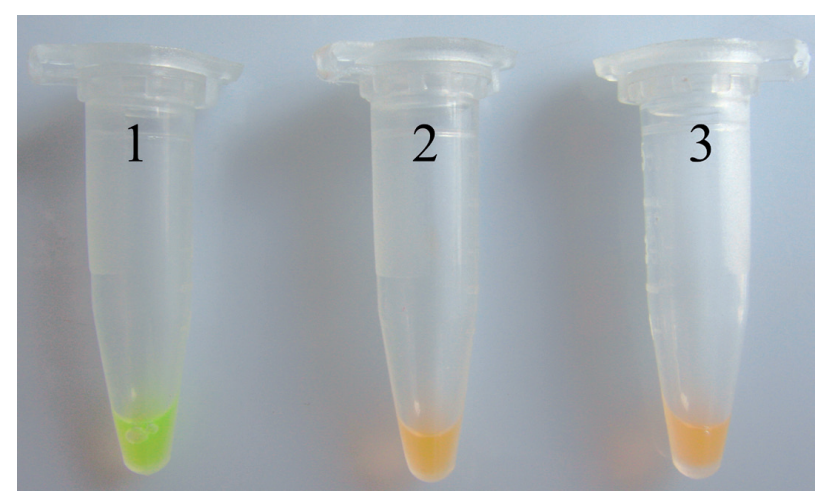

Fig. 1. Visual detection of different Toxoplasma gondii samples by LAMP method. Tube $1-T$. gondii PRU tachyzoite; Tube 2 - COS-7 cell; Tube 3 - negative control (no template).

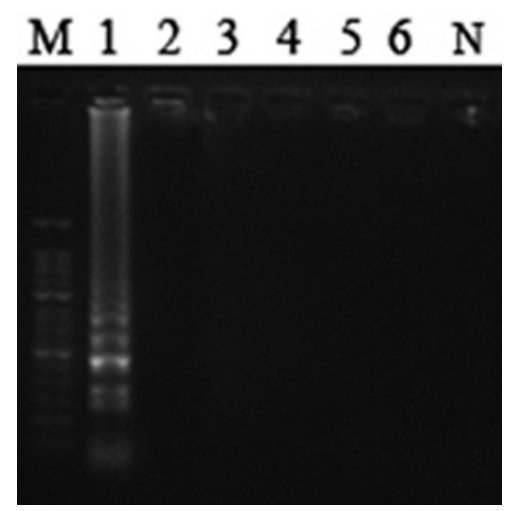

Fig. 2. B1 gene agarose gel electrophoresis product of LAMP for the detection of toxoplasmic DNA. Lane M - DNA marker; Lane 1 - Toxoplasma PRU (tachyzoites) DNA; Lane 2 - Pneumocystis jiroveci DNA; Lane 3 - Trichomonas vaginalis DNA; Lane 4 - Acanthamoeba sp. DNA; Lane 5 - Cryptosporidium parvum DNA; Lane 6 - COS-7 DNA; Lane N - negative control (no template).

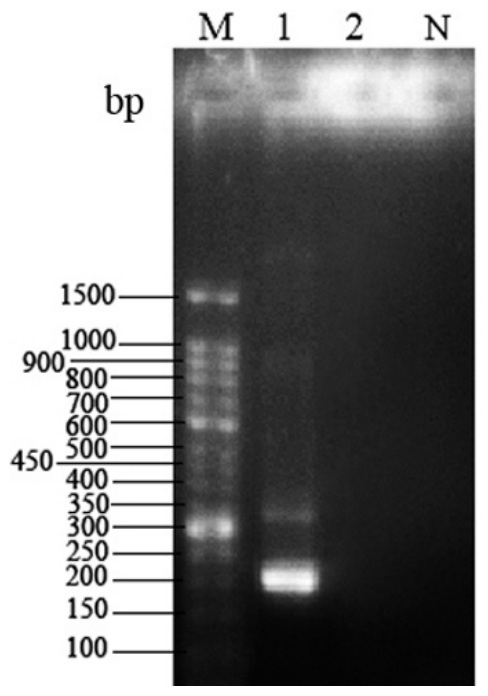

Fig. 3. Detection of different LAMP products by PCR method using LAMP outer primers. Lane M - DNA marker; Lane 1 - Toxoplasma gondii PRU tachyzoite; Lane 2 - COS-7 cell; Lane $\mathrm{N}$ - negative control (no template). 


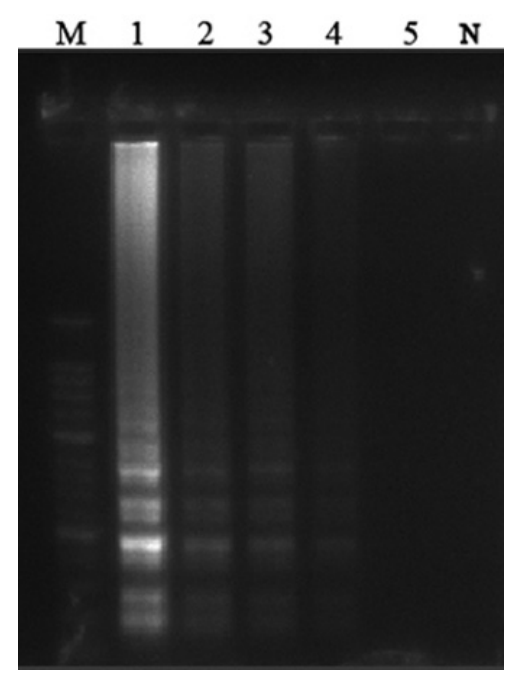

Fig. 4. The sensitivity of LAMP for the specific detection of tachyzoite toxoplasmic DNA based on the B1 gene amplification. Lane M - DNA marker; Lanes 1-5 - $1 \mathrm{ng}, 100$ pg, 10 pg, $1 \mathrm{pg}, 100 \mathrm{fg}$ of DNA; Lane $\mathrm{N}$ - negative control (no template).

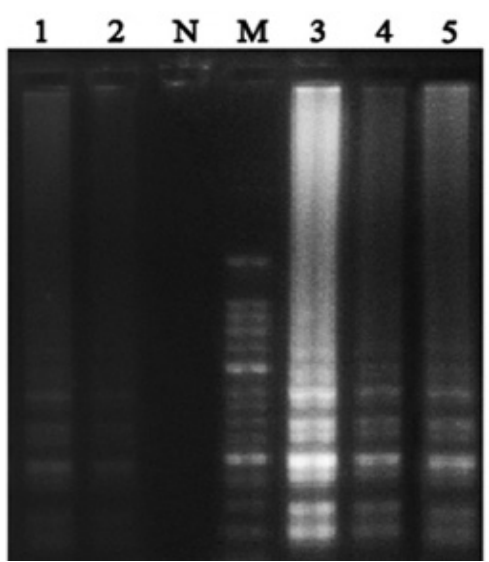

Fig. 5. Evaluation of LAMP for the detection of toxoplasmic DNA in urine samples from mice treated with dexamethasone at 90 day p.i. Lane $\mathrm{N}$ - negative control; Lane $\mathrm{M}$ - DNA marker; Lane 1-5 - DNA in urine samples 1-5.

1999). Among these techniques, nested PCR followed by hybridization has been reported to be the most sensitive method (Pujol-Rique et al. 1999). However, they are limited in low- and middle-income countries with high prevalence rates of Toxoplasma infection due to the high cost of the apparatus and long reaction time (Gross et al. 1992, Guy et al. 1996). Therefore, the development of a relatively simple, cheap and easy-to-perform test is still needed for direct detection of $T$. gondii.

It has been suggested that contrarily to PCR, LAMP is not adversely affected by irrelevant target DNA. Moreover, LAMP is able to amplify a large amount of the expected DNA under isothermal conditions in less than $1 \mathrm{~h}$, which is more rapid than conventional PCR (Notomi et al. 2000). It does not require sophisticated equipment; only a water bath or heat block is required to accomplish the whole reaction process, which is of great value for application in poorly equipped laboratories or in large-scale epidemiological studies conducted in isolated areas. The most practical characteristic of LAMP is that it allows visual detection of amplification through the addition of fluorescent dyes such as SYBR Green I (Poon et al. 2006). Based on these advantages, we conclude that LAMP is a useful and practical tool for early diagnosis of toxoplasmosis and for evaluation of chemotherapeutic effectiveness in treatment of this disease. The present study is the first to employ LAMP method to detect the Toxoplasma B1 gene from urine samples of infected mice.

Several studies have described the results obtained by LAMP with B1 (Sotiriadou and Karanis 2008), SAG1 (Krasteva et al. 2009), 529 bp gene targets (Zhang et al. 2009), or TgOWP Toxoplasma gene (Sotiriadou and Karanis 2008). The well-characterized B1 gene was chosen for the present study because this gene appears to be conserved in all $T$. gondii isolates analyzed (Ostergaard et al. 1993) and is present in at least 35 copies in the genome (Burg et al. 1989). In the present study, LAMP method was successfully established to detect B1 gene of $T$. gondii PRU strain. To determine the specificity of $T$. gondii LAMP assay, reactions were conducted for amplification of $T$. gondii DNA including non-target DNA from other parasitic protists including Pneumocystis jiroveci, Trichomonas vaginalis, Acanthamoeba sp. and Cryptosporidium parvum. As a result, only $T$. gondii DNA was amplified, whereas non-target DNA of other parasites was not amplified. Moreover, PCR was performed with two outer primers to amplify the LAMP products in order to confirm that the LAMP amplified the correct target, thereby ensuring high specificity for target amplification.

Regarding the sensitivity of LAMP, it is generally considered that LAMP is highly sensitive compared to PCR methods. In our study, the sensitivity of LAMP was evaluated by subjecting serially 10 -fold diluted DNA to LAMP. The results showed that 1 picogram (pg) DNA could be detected. Because $0.1 \mathrm{pg}$ of DNA represents approximate one $T$. gondii tachyzoite, this corresponded to 10 tachyzoites, estimated from the initial number of tachyzoites before DNA extraction and without taking into account a possible DNA loss during the extraction. The LAMP assays-demonstrated sensitivity is in agreement with that shown by Zhang et al. (2009), where the detection limits of LAMP assay (targeted at the 200- to 300-fold repetitive $529 \mathrm{bp}$ of $T$. gondii) were 10 tachyzoites. Our data indicate that the LAMP assay is sensitive enough to detect $T$. gondii DNA at a low level, and thus the use of this technique may have important clinical applications. Furthermore, this test offers the advantage of its easy accessibility by non-invasive means, such as urine sample examination. However, the LAMP sensitivity in the present assay is lower when compared to that reported by two 
groups, where the detection limit of LAMP test targeted at the B1 gene was 0.1 tachyzoite (Sotiriadou and Karanis 2008, Lau et al. 2010). These discrepancies may be attributed to many factors such as sequences of primers, concentrations of primers and other reagents in LAMP reaction, as well as temperature of incubation. Nevertheless, because our objective was to assess whether LAMP test targeted at the B1 gene can be used for detection in urine samples, we decided to perform a routine LAMP protocol without the optimized conditions, although the further optimization for conditions of the method is required.

We detected the $T$. gondii B1 gene in urine collected from experimental mice infected with PRU tachyzoites by LAMP. However, the results revealed that the B1 gene in urine was not detected on days 2 through 6 p.i. Our results are in agreement with the observation of the absence of T. gondii DNA in the urine samples of mice on day 2 through 525 after intraperitoneal inoculation with low virulence Beverley strains (Nguyen et al. 1996). It is possible that the outcome of toxoplasmosis in the mouse model is strongly dependent on experimental host species, virulence of the strain, inoculum dosage, stage of the organism, and route of inoculum administration.

In this study, ICR mice were chosen as experimental host. As a sort of outbred mice, ICR mice are more resistant to $T$. gondii. Moreover, type II strains are most common in human toxoplasmosis and as chronic infections in animals used for human consumption (Howe and Sibley 1995), we therefore used Toxoplasma PRU strain (type II strain) to infect the host.

Finally, in order to simulate the normal infection status, the mice were orally infected with the cysts of PRU strain, although the intraperitoneal infection with tachyzoites could easier induce acute infection. In our experiments, however, five mice died on day 8 through 15 p.i. The mortality rate, it seems, was inconsistent with the absence of Toxoplasma DNA in urine. One explanation is that the concentrations of $T$. gondii DNA in urine samples is below the detection limit due to the shorter survival time of infected mice resulting from strong inflammation reaction, as well as low inoculum dosage and parasite's heterogeneous distribution in tissue.

Subsequently, five mice that survived the previous infection were immunosuppressed with dexamethasone. This was because dexamethasone can induce recrudescence of toxoplasmosis in persistently infected mice to mimic the effects observed in the human AIDS population, in which $30-35 \%$ of previously infected individuals develop clinical signs of infection (Luft and Remington 1992). Immunosuppression started 90 days p.i. by adding a water-soluble form of dexamethasone to the drinking water. As a result, the B1 gene in all urine samples from five surviving mice could be detected by LAMP.

These results suggest that LAMP detection of DNA in urine may be a proper approach to the diagnosis of toxoplasmosis in chronic infection rather than early infection. Of cause, it will be necessary to assess the period of time at which the parasite or its DNA is present in urine.

In conclusion, the current study has developed a LAMP-specific protocol to amplify the T. gondii B1 DNA targeting gene. Based on these results, the LAMP amplification method might offer a valuable aid for simple, cheap, rapid and specific diagnosis of an acute toxoplasmosis, however, further research for evaluation of the method for the application in clinical diagnostics is required.

Acknowledgements. This work was supported by Social Development Program of Science and Technology Department of Zhejiang Province of China (No. 2009C33035). We declare that the experiments performed and presented in this report complied with the current laws of the People's Republic of China.

\section{REFERENCES}

Ashburn D., Davidson M.M., Joss A.W., Pennington T.H., HoYEN D.O. 1998: Improved diagnosis of reactivated toxoplasmosis. Mol. Pathol. 51: 105-109.

Burg J.L., Grover C.M., Pouletty P., Boothroyd J.C. 1989: Direct and sensitive detection of a pathogenic protozoan, Toxoplasma gondii, by polymerase chain reaction. J. Clin. Microbiol. 27: 1787-1792.

Filisetti D., Gorci M., Pernot-Marino E., Villard O., CanDOLFI E. 2003: Diagnosis of congenital toxoplasmosis: comparison of targets for detection of Toxoplasma gondii by PCR. J. Clin. Microbiol. 41: 4826-4828.

Fuentes I., Rodriguez M., Domingo C.J., del Castillo F., Juncosa T., Alvar J. 1996: Urine sample used for congenital toxoplasmosis diagnosis by PCR. J. Clin. Microbiol. 34: 2368-2371.

Germann D., Matter L. 1994: Toxoplasmosis in pregnancy. Lancet 344: 540-541.

Gross U., Roggenkamp A., Janitschke K., Heesemann J. 1992: Improved sensitivity of the polymerase chain reaction for de- tection of Toxoplasma gondii in biological and human clinical specimens. Eur. J. Clin. Microbiol. Infect. Dis. 11: 33-39.

Guan G., Chauvin A., Luo J., Inoue N., Moreau E., Liu Z., Gao J., Therisoe O.M., Ma M., Liu A., Dang Z., Liu J., Ren Q., Jin Y., Sugimoto C., Yin H. 2008: The development and evaluation of a loop-mediated isothermal amplification (LAMP) method for detection of Babesia spp. infective to sheep and goats in China. Exp. Parasitol. 120: 39-44.

Guy E.C., Pelloux H., Lappalainen M., Aspock H., Hassl A., Melby K.K., Holberg-Pettersen M., Petersen E., Simon J., Ambroise-Thomas P. 1996: Interlaboratory comparison of polymerase chain reaction for the detection of Toxoplasma gondii DNA added to samples of amniotic fluid. Eur. J. Clin. Microbiol. Infect. Dis. 15: 836-839.

Homan W.L., Vercammen M., De Braekeleer J., Verschueren H. 2000: Identification of a 200 - to 300 -fold repetitive $529 \mathrm{bp}$ DNA fragment in Toxoplasma gondii, and its use for diagnostic and quantitative PCR. Int. J. Parasitol. 30: 69-75. 
Howe D.K., Sibley L.D. 1995: Toxoplasma gondii comprises three clonal lineages: correlation of parasite genotype with human disease. J. Infect. Dis. 172: 1561-1566.

Huskinson J., Stepick-Biek P., Remington J.S. 1989: Detection of antigens in urine during acute toxoplasmosis. J. Clin. Microbiol. 27: 1099-1101.

Jannuzzi C., Canessa A., Spandonari M., Terragna A. 1981: Evaluation of the dye test, IgM-IFAT, and ELISA for the diagnosis of toxoplasmic infection in pregnancy. Int. J. Biol. Res. Pregnancy 2: 123-127.

Johnson J.D., Butcher P.D., Savva D., Holliman R.E. 1993: Application of the polymerase chain reaction to the diagnosis of human toxoplasmosis. J. Infect. 26: 147-158.

Krasteva D., Toubiana M., Hartati S., Kusumawati A., DuBRemetz J.F., Widada J.S. 2009: Development of loop-mediated isothermal amplification (LAMP) as a diagnostic tool of toxoplasmosis. Vet. Parasitol. 162: 327-331.

Krick J.A., Remington J.S. 1978: Toxoplasmosis in the adult - an overview. N. Engl. J. Med. 298: 550-553.

Lau Y.L., Meganathan P., Sonaimuthu P., Thiruvengadam G., Nissapatorn V., Chen Y. 2010: Specific, sensitive, and rapid diagnosis of active toxoplasmosis by a loop-mediated isothermal amplification method using blood samples from patients. J. Clin. Microbiol. 48: 3698-3702.

Lee P.Y., Mangan J., Holliman R.E., Butcher P.D. 1999: Quantitation of Toxoplasma gondii DNA in a competitive nested polymerase chain reaction. J. Clin. Pathol. 52: 61-64.

Luft B.J., Remington J.S. 1988: AIDS commentary. Toxoplasmic encephalitis. J. Infect. Dis. 157: 1-6.

Luft B.J., Remington J.S. 1992: Toxoplasmic encephalitis in AIDS. Clin. Infect. Dis. 15: 211-222.

Mondesire R.R., Charlton D.E., Tizard I.R. 1981: A standardized enzyme-linked immunosorbent assay (ELISA) for the detection of antibodies to Toxoplasma gondii. J. Immunoassay 2: 45-57.

Nguyen T.D., de Kesel M., Bigaignon G., Hoet P., Pazzaglia G., Lammens M., Delmee M. 1996: Detection of Toxoplasma gondii tachyzoites and bradyzoites in blood, urine, and brains of infected mice. Clin. Diagn. Lab. Immunol. 3: 635-639.

Nitru Z.K., Mikosza A.S., Matovu E., Enyaru J.C., Ouma J.O., Kibona S.N., Thompson R.C., Ndung'u J.M. 2008: African trypanosomiasis: sensitive and rapid detection of the subgenus Trypanozoon by loop-mediated isothermal amplification (LAMP) of parasite DNA. Int. J. Parasitol. 38: 589-599.

Notomi T., Okayama H., Masubuchi H., Yonekawa T., Watanabe K., Amino N., Hase T. 2000: Loop-mediated isothermal amplification of DNA. Nucleic Acids Res. 28: e63.

Ostergaard L., Nielsen A.K., Black F.T. 1993: DNA amplification on cerebrospinal fluid for diagnosis of cerebral toxoplas-

Received 4 August 2011 mosis among HIV-positive patients with signs or symptoms of neurological disease. Scand. J. Infect. Dis. 25: 227-237.

Poon L.L., Wong B.W., Ma E.H., Chan K.H., Chow L.M., Abeyewickreme W., Tangpukdee N., Yuen K.Y., Guan Y., Looareesuman S., Peiris J.S. 2006: Sensitive and inexpensive molecular test for falciparum malaria: detecting Plasmodium falciparum DNA directly from heat-treated blood by loop-mediated isothermal amplification. Clin. Chem. 52: 303-306.

Pujol-Rique M., Derouin F., Garcia-Quintanilla A., Valls M.E., Miro J.M., Jimenez D.A.M. 1999: Design of a one-tube hemi-nested PCR for detection of Toxoplasma gondii and comparison of three DNA purification methods. J. Med. Microbiol. 48: 857-862.

Remington J.S., McLeod R., Desmonts G. 1995: Toxoplasmosis. In: J.S. Remington and J.O. Klein (Eds.), Infectious Diseases of the Fetus and New-born Infants. 4th Ed., W.B. Saunders, Philadelphia, pp. 140-267.

Ruskin J., Remington J.S. 1976. Toxoplasmosis in the compromised host. Ann. Intern. Med. 84: 193-199.

Saeij J.P.J., Arrizabalaga G., Boothroyd J.C. 2008: A cluster of four surface antigen genes specifically expressed in bradyzoites, SAG2CDXY, plays an important role in Toxoplasma gondii persistence. Infect. Immun. 76: 2402-2410.

Sotiriadou I., Karanis P. 2008: Evaluation of loop-mediated isothermal amplification for detection of Toxoplasma gondii in water samples and comparative findings by polymerase chain reaction and immunofluorescence test (IFT). Diagn. Microbiol. Infect. Dis. 62: 357-365.

Stagno S. 1980: Congenital toxoplasmosis. Am. J. Dis. Child. 134: 635-637.

Uemura N., Makimura K., Onozaki M., Otsuka Y., Shibuya Y., Yazaki H., Kikuchi Y., Abe S., Kudoh S. 2008: Development of a loop-mediated isothermal amplification method for diagnosing Pneumocystis pneumonia. J. Med. Microbiol. 57: $50-57$

YANG Q.L., Zhang R.S., Wu H.P. 2008: Detection of Toxoplasma gondii DNA by loop-mediated isothermal amplification. Zhongguo Ji Sheng Chong Xue Yu Ji Sheng Chong Bing Za Zhi 26: 304-306. (In Chinese, with English abstract.)

Zangerle R., Allerberger F., Pohl P., Fritsch P., Dierich M.P. 1991: High risk of developing toxoplasmic encephalitis in AIDS patients seropositive to Toxoplasma gondii. Med. Microbiol. Immunol. 180: 59-66.

Zhang H., Thekisoe O.M., Aboge G.O., Kyan H., Yamagishi J., Inoue N., Nishikawa Y., Zakimi S., Xuan X. 2009: Toxoplasma gondii: sensitive and rapid detection of infection by loop-mediated isothermal amplification (LAMP) method. Exp. Parasitol. 122: 47-50.

Accepted 11 December 2011 